\title{
A Multi-Band Body-Worn Distributed Exposure Meter for Personal Radio-Frequency Dosimetry in Diffuse Indoor Environments
}

\author{
Reza Aminzadeh, Student Member, IEEE, Arno Thielens, Davy Paul Gaillot, Martine Liénard, Lamine Koné, \\ Sam Agneessens, Patrick Van Torre, Matthias Van den Bossche, Leen Verloock, Stefan Dongus, Marloes Eeftens, \\ Anke Huss, Roel Vermeulen, René de Seze, Elisabeth Cardis, Hendrik Rogier, Senior Member, IEEE, \\ Martin Röösli, Luc Martens, Member, IEEE, and Wout Joseph, Senior Member, IEEE
}

\begin{abstract}
A multi-band body-worn distributed exposure meter (BWDM) is designed and calibrated for diffuse fields in a reverberation chamber (RC) for personal exposure assessment in indoor environments. The BWDM uses 22 nodes distributed over the torso and measures the incident power density $\left(S_{i n c}\right)$ on body for 11 telecommunication bands in the frequency range 790-5513 MHz. In order to calibrate the measurement device in diffuse fields, a protocol is proposed for on-body calibration of the BWDM. This protocol is applicable to wearable personal exposure meters in general. The BWDM and the proposed calibration protocol are validated in five indoor locations and five frequency bands (the downlink bands at 800, 900, 1800 and $2100 \mathrm{MHz}$ and WiFi $2 \mathrm{GHz}$ ) using a tri-axial broadband antenna and a spectrum analyzer (SA). The calibration shows that the BWDM has a relatively low measurement uncertainty with a $68 \%$ confidence interval on its antenna apertures, in the range 3.4-5.5 dB. A maximum difference of $0.9 \mathrm{~dB}$ is obtained for the total exposure in the test areas between the measurements of the BWDM and SA, which is an excellent agreement.
\end{abstract}

Index Terms-Wearable, Radio-frequency, Personal exposure, Dosimetry, Indoor, Reverberation chamber

\section{INTRODUCTION}

This work was supported by the Research Foundation Flanders (FWO) under grant agreement No G003415N and the French National Research Program for Environmental and Occupational Health of ANSES (2015/2 $\mathrm{RF} / 07$ ) as part of project ACCEDERA. A.T. has received funding from the European Union's Horizon 2020 research and innovation programme under the Marie Skłodowska-Curie grant agreement No 665501 with the FWO (Corresponding author: Reza Aminzadeh)

R. Aminzadeh, A. Thielens, S. Agneessens, P. Van Torre, M. V. d. Bossche, L. Verloock, H. Rogier, L. Martens and W. Joseph are with the Department of Information Technology (INTEC), Ghent University/imec, TechnologieparkZwijnaarde 126, B-9052, Ghent, Belgium (Email: reza.aminzadeh@ugent.be)

A. Thielens is with Berkeley Wireless Research Center, Department of Electrical Engineering and Computer Sciences, University of California, Berkeley, CA 94704, United States.

D.P. Gaillot, M. Liénard and L. Koné are with the University of Lille, IEMN-TELICE, Villeneuve d'Ascq, France.

S. Dongus, M. Eeftens and M. Röösli are with the Department of Epidemiology and Public Health, Swiss Tropical and Public Health Institute, Socinstrasse 57, Basel 4051, Switzerland.

S. Dongus, M. Eeftens and M. Rööli are with the University of Basel, Petersplatz 1, 4051 Basel, Switzerland.

A. Huss and R. Vermeulen are with the Institute for Risk Assessment Sciences (IRAS), Utrecht University, Utrecht, the Netherlands.

R.d. Seze is with the TOXI/PERITOX UMR I-01 unit, National Institute for Industrial Environment and Risks (INERIS), Verneuil en Halatte, France.

E. Cardis is with the Barcelona Institute for Global Health (ISGlobal), Barcelona, Spain.
C HARACTERIZATION of human exposure to radiofrequency (RF) electromagnetic fields (EMFs) is identified by the world health organization (WHO) as an important issue to be addressed by several countries [1]. Recently, potential adverse effects of mobile phone use on brain functions such as figural memory has been reported [2]. Additionally, thermal effects of RF-EMFs are well established [3]. In order to avoid these thermal effects of human exposure to RF-EMFs, the field strengths or alternatively power densities incident on the body $\left(S_{i n c}\right)$ are limited by adhering to reference levels such as those issued by the International Commission on NonIonizing Radiation Protection (ICNIRP) [3]. Personal exposure meters (PEMs) are commonly used to measure individual exposure to RF radiation [4]. These portable devices provide continuous monitoring of RF-EMFs in different frequency bands at the same location for the subject wearing the device. Measurement protocols have been proposed for the use of PEMs [5]-[7]. However, PEMs measurements are associated with large measurement uncertainties due to presence of wearer's body e.g. body shielding [8]. Thus, PEMs measure the electric fields on the body instead of the actual incident fields, which results in uncertainties on the estimation of the actual fields [9]-[11]. In order to address these issues, on-body calibration of PEMs and the use of multiple PEMs have been proposed in the literature [9], [11], [12]. Additionally, singleband [13]-[15] and a multi-band [16] body-worn distributed exposure meter (BWDM) have been proposed using on-body calibration in an anechoic chamber to increase the measurement accuracy of incident fields on-body. This calibration has proven effective for outdoor environments, where the incident RF-EMFs can be considered as specular components [17].

However, people spend more than $80 \%$ of their time indoors [18]. The increasing number of indoor wireless technologies such as smart home gadgets, makes it crucial to assess human exposure to RF-EMFs in indoor environments. The total power in an indoor environment is composed of specular and diffuse multi-path components (DMC) [19], [20]. The former is a result of coherent reflection (e.g. large surfaces like walls) whereas the latter results from non-coherent reflections (due to the presence of different objects) in an indoor area. In an indoor environment, DMC may contribute up to $95 \%$ to the total incident power density [21] and $88 \%$ to the whole-body averaged specific absorption rate [22]. In order 
to assess human exposure to RF-EMFs in diffuse fields, an on-body calibration of PEMs in such fields was recommended in [12]. Reverberation chambers (RC) are environments in which diffuse fields can be generated in a controlled manner [23]. Previously, the response of two commercial PEMs has been determined using on-body calibration in a RC [12]. The correction factor of the calibrated (two) PEMs in the $\mathrm{RC}$ (diffuse fields) was found to be up to 4.8 times lower compared to when the PEMs are calibrated on-body in an anechoic chamber (specular fields) [12]. In other words, the calibrated PEMs in an anechoic chamber underestimate the diffuse exposure up to a factor larger than 4. This is a strong indication that a separate calibration of PEMs in a $\mathrm{RC}$ is necessary. However, on the one hand, a consistent onbody calibration protocol for PEMs/BWDM in diffuse fields is missing in the literature. On the other hand, to the best of authors' knowledge, no BWDM has been proposed or demonstrated in literature that is able to measure diffuse fields in indoor environments. It is expected that also in diffuse fields a BWDM will have a lower measurement uncertainty than commercial PEMs.

This paper presents, for the first time, the on-body calibration of the first BWDM for diffuse fields in a reverberation chamber (RC). The goal is to use the BWDM to simultaneously measure personal exposure to RF fields in indoor diffuse environments. First, the BWDM is designed and optimized based on the calibration measurements on a test person in an anechoic chamber. Second, the optimized BWDM is calibrated on the same person in a RC to determine its measurement uncertainty. In addition, a protocol is proposed for calibration of personal exposure meters (including conventional PEMs and personal distributed exposure meters) in a $\mathrm{RC}$ for measurement of personal exposure in diffuse indoor environments. The results are validated by performing a real measurement campaign in an indoor office environment.

\section{MAterials AND Methods}

Section II-A describes the on-body setup and design of the BWDM. Section II-B introduces the used PEM, the ExpomRF. Section II-C presents the on-body calibration while Section II-D explains the cross-talk. The validation measurements are presented in Section II-E.

\section{A. $B W D M$}

The BWDM comprises 22 nodes connected to a common serial bus system. A portable master unit (see Fig. 1) controls the bus communication and can be connected to a laptop computer to read/erase measurement data. The master unit monitors the correct operation and synchronization of the nodes. The user can synchronously record time-stamps in all the 22 nodes by pressing a button on the master unit. The 22 nodes were placed on 22 locations (A to $\mathrm{V}$ ) on the front and back of the body torso as well as the hips as shown in Fig. 1(a). The arms, the legs and the head were excluded for antenna deployment since their movements during the measurements would influence the performance of antennas [24]. Additionally, placing the used antennas on the head and limbs might impede the subject's movements, which might negatively impact potential measurements done with the device. The BWDM covers 11 frequency bands allocated to legacy wireless communication systems from $800 \mathrm{MHz}$ to $6 \mathrm{GHz}$ as listed in Table I. For each frequency band, two nodes were considered on the front and back of the body to compensate for shielding of the body [11], [16]. The optimized location and polarization of the nodes (see Table I) were determined by on-body calibration of the BWDM on a test person (male; height: $183 \mathrm{~cm}$ ) in an anechoic chamber [16]. At each center frequency, the term optimized was defined as the location and the polarization that provided the minimum measurement uncertainty. The polarizations in Table I are relative to the ground floor: vertical (V) polarization is orthogonal to the floor and horizontal $(\mathrm{H})$ is parallel to the floor.

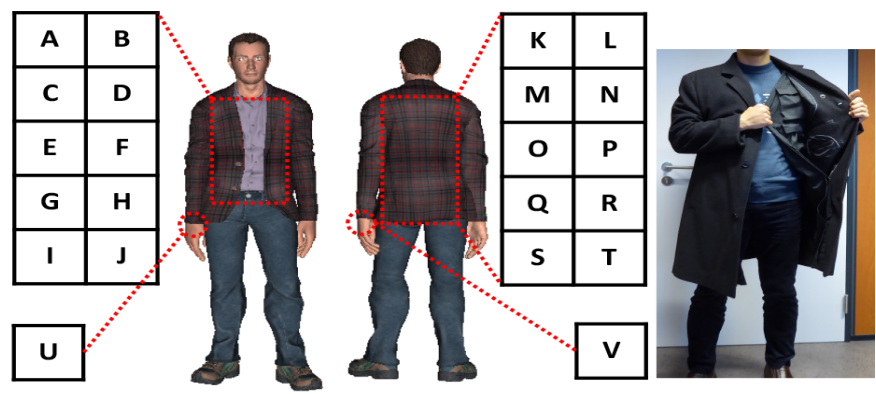

(a)
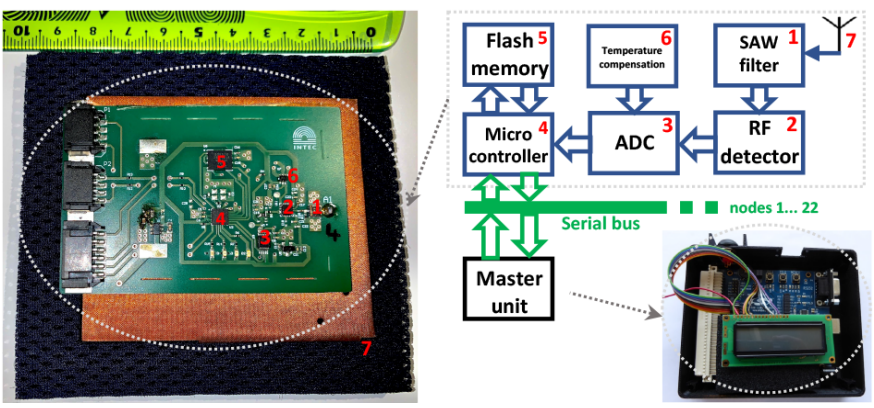

(b)

Fig. 1. (a) Location of the nodes on body (left) integrated into a garment (right). (b) Prototype of a node (left) and the master unit (bottom right).

1) antennas: The nodes are composed of linearly (WiFi$5 \mathrm{G}$ ) and elliptically (for the other bands) polarized textilecompatible substrate-integrated-waveguide (SIW) antennas and compact receiver electronics to register the incident EMFs. The design of the antennas is presented in [25]. The conductive parts and the antenna substrate were made from copper-coated nylon and closed-cell expanded rubber commonly found in garments of firefighters, respectively. $\mathrm{A} \mathrm{CO}_{2}$ laser with a spot size smaller than $0.1 \mu \mathrm{m}$ was used to pattern the textile materials. The antennas have a power reflection coefficient lower than $-10 \mathrm{~dB}$ in the desired frequency bands (cf. Table I). Compared to [15], the BWDM antennas have high-body antenna isolation and are more sensitive to radiation incident from directions away from the human body. The antennas have a gain in the 3-5 $\mathrm{dBi}$ range (along the direction away from the body). The antenna polarization is linear with an axial ratio 
between 5 and $15 \mathrm{~dB}$. The smallest and the largest antennas have a surface of $9 \times 10 \mathrm{~cm}^{2}$ and $11 \times 11 \mathrm{~cm}^{2}$, respectively.

2) receiver nodes: Figure 1(b) shows a prototype of the designed nodes for the BWDM. The receiving nodes are based on logarithmic radio-frequency (RF) detector chips, preceded by suitable band-pass filters for each band of interest. Ceramic Surface Acoustic Wave (SAW) filters are employed, providing a large out-of-band rejection in one small Surface Mounted Device (SMD). Concerning the detector chips, for the $5.8 \mathrm{GHz}$ band the AD8318 is used, whereas for all the lower frequency bands, the ADL5513 is employed. Both detector chips are precision circuits manufactured by Analog Devices Inc. The ADL5513 provides a dynamic range of $80 \mathrm{~dB}$, but its bandwidth is limited to $4 \mathrm{GHz}$. The AD8318 has an $8 \mathrm{GHz}$ bandwidth but a more limited dynamic range of $55 \mathrm{~dB}$. The temperature-dependent offset of the logarithmic power detector is compensated automatically using a Digitalto-analog-converter (AD5641, Analog Devices Inc) connected to the central micro-controller with a built-in temperature sensor. In the construction of the nodes on Printed Circuit Board (PCB), standard FR4 board is used for all bands up to $2.45 \mathrm{GHz}$. For the $5.8 \mathrm{GHz}$ band, FR4 material causes too much signal loss and therefore the more expensive Rogers $0.5 \mathrm{~mm}$ thick Isola I-tera high frequency laminate was chosen.

The output of both detectors is a voltage proportional to the logarithm of the input power. This voltage is sampled by a 16-bit Analog-to-Digital Converter (ADC), connected to the central micro-controller via the Serial Peripheral Interface (SPI). The micro-controller performs a first processing of the measurements in order to determine the maximum, minimum and average power over (100 samples per second) each onesecond measurement interval. Both the arithmetic and the geometric averages are calculated by the micro-controller, resulting in four measurement values to be stored in the 32 MBit local flash memory unit each second. The current consumption of each node and the BWDM (including 22 nodes and the master unit) in full operation are $39 \mathrm{~mA}$ and $1200 \mathrm{~mA}$ at $5 \mathrm{~V}$, respectively. A $5 \mathrm{~V}(13000 \mathrm{mAh})$ battery powers the BWDM. The minimum and maximum weight of the nodes are $15 \mathrm{~g}$ and $40 \mathrm{~g}$, respectively.

TABLE I

LOCATION AND ORIENTATION OF THE NODES ON BODY AS WELL AS THEIR FREQUENCY BANDS.

\begin{tabular}{cccc}
\hline Band name & Range $(\mathrm{MHz})$ & $\mathrm{F}_{c}(\mathrm{MHz})$ & Location \\
\hline \hline 800-DL & $790-821$ & 806 & $C^{H}, Q^{V}$ \\
900-UL & $879-915$ & 896 & $I^{V}, O^{H}$ \\
900-DL & $921-960$ & 941 & $G^{H}, K^{V}$ \\
1800-UL & $1710-1785$ & 1748 & $A^{V}, S^{H}$ \\
1800-DL & $1805-1880$ & 1843 & $D^{H}, R^{V}$ \\
DECT & $1880-1900$ & 1890 & $H^{H}, L^{V}$ \\
2100-UL & $1900-1980$ & 1940 & $F^{V}, P^{H}$ \\
2100-DL & $2110-2170$ & 2140 & $U^{H}, V^{V}$ \\
WiFi-2G & $2400-2485$ & 2443 & $J^{V}, N^{H}$ \\
2600-DL & $2620-2690$ & 2655 & $B^{H}, T^{V}$ \\
WiFi-5G & $5150-5875$ & 5513 & $E^{H}, M^{V}$ \\
\hline
\end{tabular}

$\mathrm{F}_{c}$ : center frequency; $\mathrm{V}$ : vertical polarization; $\mathrm{H}$ : Horizontal polarization.

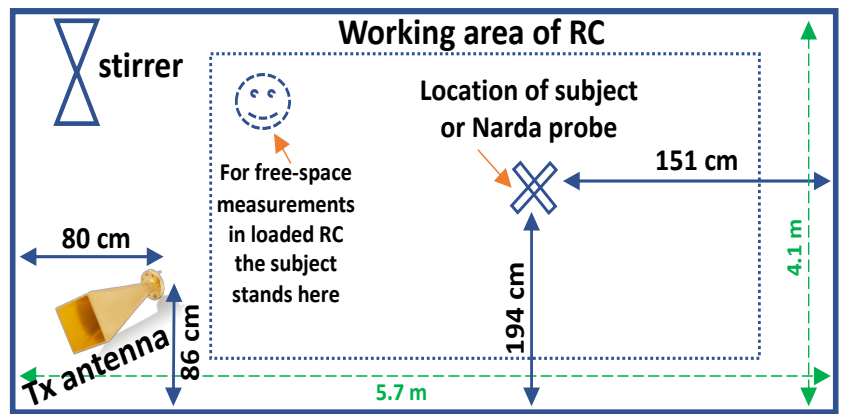

Fig. 2. The setup for calibration measurements in the reverberation chamber (top view).

\section{B. ExpoM-RF}

A conventional PEM, ExpoM-RF (Fields at Work GmbH, Zürich, Switzerland) was used in this study and is denoted as Expom in this paper. The Expom has a detection limit of $0.024 \mu \mathrm{W} / \mathrm{m}^{2}$ for 2100-UL, 2100-DL and 2600-DL. The detection limit is $6.6 \mu \mathrm{W} / \mathrm{m}^{2}$ for Wireless Fidelity $5 \mathrm{GHz}$ (WiFi-5G) and is $0.07 \mu \mathrm{W} / \mathrm{m}^{2}$ for other studied frequency bands (see Table I).

\section{Calibration measurements}

We present a procedure for on-body calibration of PEMs, including the BWDM, in a RC. The calibration measurements consist of two types of measurements: on body and in offbody. Figure 2 illustrates the setup for calibration measurements in the RC. The room dimensions are $5.7 \times 4.1 \times 2.8 \mathrm{~m}^{3}$. The different electromagnetic modes in the RC were excited using a stirrer composed of four rotating metallic sheets. The $\mathrm{RC}$ has a total field standard deviation below $1.5 \mathrm{~dB}$ (below the $3 \mathrm{~dB}$ tolerance limit) in the range of $800-1000 \mathrm{MHz}$ and decreases with increasing frequency. An SAS-571 horn antenna (TX) with vertical polarization, operating in the range of studied frequency bands was used. The TX emited a constant power of $1 \mathrm{~mW}$ at each center frequency as listed in Table I. All the measurements were performed in the working area of the RC (dashed lines in Fig. 2) where fields had uniform distribution [26].

1) Off-body measurements: A broadband field meter NBM550 in combination with EF 0391/EF 0691 probes (NARDA Safety Solutions) was placed in the working area of the RC (See Fig. 2). The goal was to measure the incident power density $S_{i n c}^{o f b}$ in the RC. The NBM-550 broadband meter in combination with $\mathrm{EF} 0391$ and $\mathrm{EF} 0691$ probes has a combined standard uncertainty of $1.1 \mathrm{~dB}$ (including frequency sensitivity, linearity, thermal and isotropic responses) on the off-body measurements. $S_{\text {inc }}^{\text {ofb }}$ was measured for two scenarios: unloaded RC (in absence of the test person) and loaded RC (in presence of the test person).

To keep the variation of the RC's Q-factor to minimum [27], it was suggested to keep the subject in the chamber when performing the off-body measurements in a RC. This keeps the Q-factor of the RC and consequently the incident power density constant for all measurements including on-body and 
in off-body. For off-body measurements, the test person stood in the working area but far away from the broadband field meter in order not to influence the measured power densities (probe's performance).

At each center frequency, the incident power density was measured at different heights in the range of $68.5-201 \mathrm{~cm}$ from the floor. During a full rotation of the stirrer (at a speed of $\left.10^{\circ} / \mathrm{s}\right), 36$ samples were measured at a sample rate of $1 \mathrm{~Hz}$. The measurements were averaged over the height and the positions of the stirrer per frequency band. This was repeated for the unloaded (empty) and the loaded (with person) chamber.

2) On-body calibration of BWDM: The test person, for whom the BWDM was optimized (for details refer to [16]), was placed in the $\mathrm{RC}$ at the same location as the broadband field meter, in standing posture and wearing the BWDM. At each center frequency, the received power $P_{r}$ was recorded simultaneously on two nodes (on the front and back), at a sample rate of $1 \mathrm{~Hz}$, for a full rotation of the stirrer. The stirrer rotated at $2 \%$ sesulting in 180 received $P_{r}$ samples on each node as a function of the rotation angle $\varphi$ of the stirrer. Compared to off-body measurements, the rotation speed decreased to register more samples to ensure that the number of samples are sufficient for correct estimation of the measurement uncertainty. These received powers $P_{r}$ were first geometrically averaged over the two nodes on the front and back (in each frequency band i) $P_{r, i, g e o m}$ and then were averaged over $\varphi$, in order to determine the on-body geometrically averaged antenna aperture (AA) over front and back in diffuse fields:

$$
A A_{\text {geom }, i}=\frac{\left\langle P_{r, i, g e o m}\right\rangle}{S_{i n c, i}^{o f b}},
$$

where $i$ is the center frequency of the eleven studied frequency bands and \langle\rangle denotes the average over $\varphi$ of the stirrer. During the measurements in a real environment, the BWDM registers $P_{r, i}^{\text {meas }}$ on each node. These data were then converted into measured power densities incident on body $S_{i n c, i}^{m e a s}$ using geometrically averaged $A A_{\text {geom }, i}$ :

$$
S_{\text {inc }, i}^{\text {meas }}=\frac{P_{r, i, g e o m}^{\text {meas }}}{A A_{\text {geom }, i}} .
$$

\section{Cross-talk}

Cross-talk is the fraction of power registered $\left(P_{r}\right)$ in a specific frequency band $j$ while the emission occurs in another frequency band $i$ (misclassification). The ideal cross-talk is a unit matrix, of size $n$ ( $n$ : number of frequency bands) with ones on the main diagonal and zeros else where. In this paper cross-talk $C_{i j}$ for diffuse fields is defined as:

$$
C_{i j}=\frac{\left\langle P_{r, j}\left(f_{i}\right)\right\rangle}{\left\langle P_{r, i}\left(f_{i}\right)\right\rangle}
$$

For the BWDM, nodes $\mathrm{A}$ to $\mathrm{J}$ and $\mathrm{U}$ (one node per frequency band) were placed on front of the body (see Fig. 1(a)). To compare the on-body cross-talk of BWDM with commercial PEMs, an Expom was worn simultaneously on the left hip of the subject (above the location of node V) during the measurements in the RC. The received power $P_{r}$ for each frequency band was averaged over all positions of the stirrer. For the Expom, $P_{r}$ in Eq. 3 is replaced by $\left(E_{r}\right)^{2}$ where $E_{r}$ is the electric field registered by the Expom.

\section{E. Validation in a real indoor environment}

The goal of this validation was to assess the applicability of the proposed calibration procedure for RF exposure measurements in real indoor environments. Therefore, using the $A A$ values determined from unloaded and loaded $\mathrm{RC}$, the measured $S_{i n c}$ values by the BWDM in an indoor office environment (see Fig. 3) were compared with the measured $S_{i n c}$ of an isotropic field probe at the same locations (the golden standard in RF-EMF exposure measurements [28]). This measurement setup has an uncertainty of $\pm 3 \mathrm{~dB}$ for the electric fields [28], [29]. Five measurement points including two office rooms and three spots in the corridor were considered as shown in Fig. 3.

Five downlink (DL) bands which are dominant (present) in indoor areas were considered here: 800, 900, 1800 and $2100 \mathrm{MHz}$ as well as $\mathrm{WiFi}-2 \mathrm{G}$. The $S_{i n c}$ was measured using an isotropic, tri-axial antenna and a spectrum analyzer (FSL, Rhode \& Schwartz, Germany). The height of the antenna was $120 \mathrm{~cm}$ above the ground on each of the five locations. The spectrum analyzer (SA) measured in continuous sweep mode using a root-mean-squared (RMS) detector with a resolution bandwidth of $300 \mathrm{kHz}$. For each frequency band, 501 points were considered which resulted in a sweep time of $300 \mathrm{~ms}$. Static measurements were chosen due to the lower measurement uncertainty compared to the dynamic measurements. Moreover, measurements of the isotropic antenna are not impeded by the operator's body that moves the antenna. Following the measurement at each location, the test person, who was wearing the BWDM, walked around in a square of $1 \times 1 \mathrm{~m}^{2}$ centered on each of the five points for 15 minutes. This is due to the fact that the on-body calibration considers a dynamic environment with changing incident fields. Therefore, static measurements using the BWDM do not make sense in this case.

\section{RESUlTS AND DISCUSSION}

Section III-A presents the results of on-body calibrations. Validation measurements are discussed in Section III-C and the cross talk of BWDM is compared with the Expom in Section III-B.

\section{A. On-body calibration of the BWDM}

Figure 4 illustrates the $68 \%$ confidence interval $\left(\mathrm{CI}_{68}\right)$ of the on-body antenna aperture (AA) in diffuse fields for 22 nodes of the the single body-worn (front and back) as well as the geometric average over each pair of nodes in 11 frequency bands. This figure shows that using two nodes on the front and back of the body reduces the $\mathrm{CI}_{68}$ in diffuse fields (black bars versus grey bars).

For the nodes on the front, the minimum and maximum $\mathrm{CI}_{68}$ are $8.8 \mathrm{~dB}(900-\mathrm{DL})$ and $4 \mathrm{~dB}(\mathrm{WiFi}-5 \mathrm{G})$, respectively. For the 


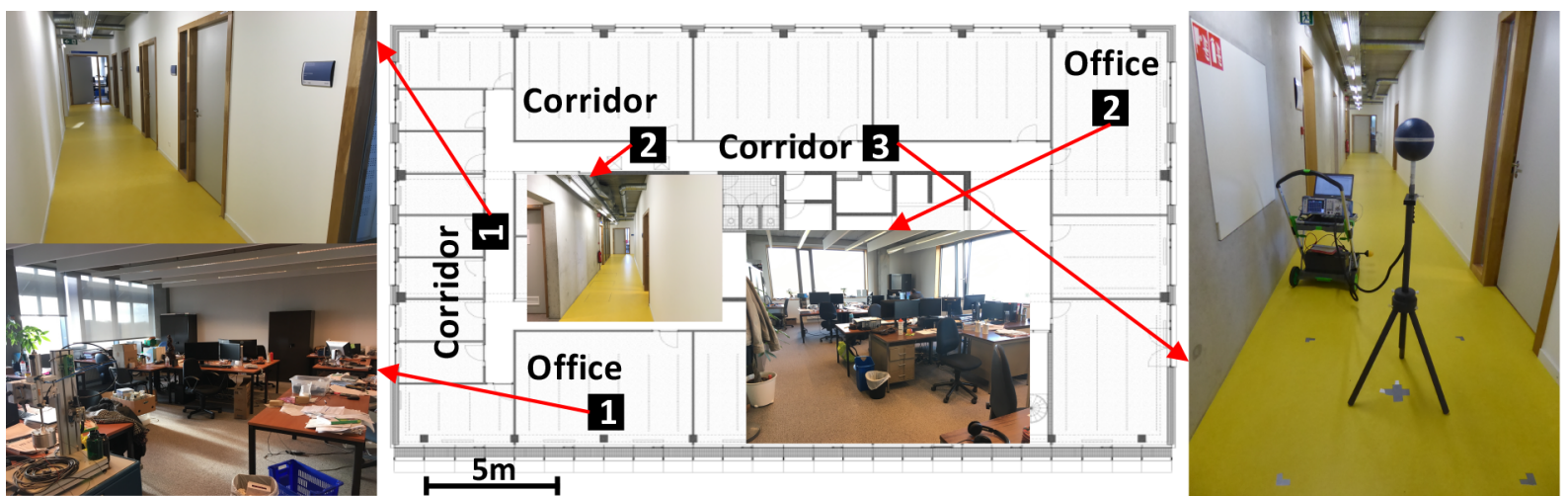

Fig. 3. Locations of measurement points (black squares) used for validation measurements in an indoor office environment. The measurement setup with SA is shown on the right side.

nodes on the back, $900-\mathrm{DL}$ and $2100-\mathrm{DL}$ have the highest $\mathrm{CI}_{68}$ $(9 \mathrm{~dB})$ while $\mathrm{WiFi}-5 \mathrm{G}$ has the lowest $\mathrm{CI}_{68}(5 \mathrm{~dB})$. The value of $\mathrm{CI}_{68}$ is $3.4 \mathrm{~dB}$ to $5.5 \mathrm{~dB}$ for the geometric average over two nodes on the front and back. This is an improvement of $3.3 \mathrm{~dB}$ for the nodes on the front (900-DL) and $3.5 \mathrm{~dB}$ for the nodes on the back (900-DL and 2100-UL) in comparison to single antennas. Similarly, a $\mathrm{CI}_{68}$ in the range of $3.3 \mathrm{~dB}$ (2100-DL) to $5.7 \mathrm{~dB}$ (2600-DL) was obtained for the BWDM calibrated on the same subject in anechoic chamber (subject \#Sb-1 in [16]).

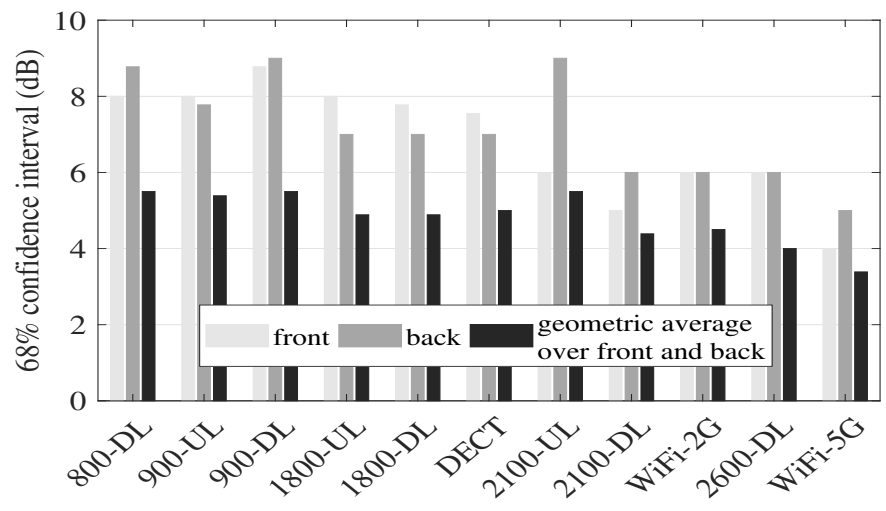

Fig. 4 . The $68 \%$ confidence interval of the on-body antenna aperture in diffuse fields for 22 nodes in 11 frequency bands.

Figure 5 depicts the same $68 \%$ confidence interval $\left(\mathrm{CI}_{68}\right)$ of the on body antenna aperture (AA) in diffuse fields for different configurations of two antennas on the body in four frequency bands (900-DL, 900-UL, WiFi-2G and 2600-DL). All considered configurations are pairs of locations on the front and back of the body (or two-opposite hips). For 900UL, 900-DL and WiFi-2G changing the locations of the nodes (see Fig. 1(a)) from IO to EK, GK to $\mathrm{BN}$ and $\mathrm{JN}$ to GM, changes $\mathrm{CI}_{68}$ by $0.1 \mathrm{~dB}, 0.5 \mathrm{~dB}$ and $0.2 \mathrm{~dB}$, respectively. The $\mathrm{CI}_{68}$ remains constant for 2600-DL in Fig. 5 by changing the location of the nodes from BT to DL. This shows that the location of the two nodes on the body has little to no influence on the $\mathrm{CI}_{68}$, if two locations on the front and back are chosen. Hence, the optimized locations of the nodes for the BWDM calibrated in anechoic chamber [16] can be used in the reverberation chamber. Since the polarization of the incident waves in the RC has uniform distribution [19], changing the polarization of the nodes does not change the results.

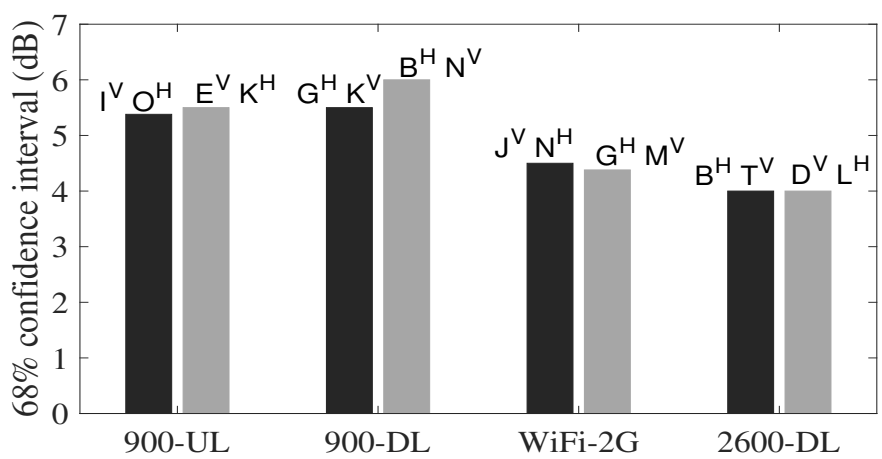

Fig. 5. The $68 \%$ confidence interval of the on-body antenna aperture in diffuse fields for different positions on body. The results are obtained for the geometric average over two nodes on the front and back of the body per band/position (letters above the bars) in the RC. The polarization of the nodes $(\mathrm{V} / \mathrm{H})$ are those obtained from calibrations in the anechoic chamber [16].

Table II lists the AA values for each frequency band obtained from on-body calibrations in the RC. For the unloaded $\mathrm{RC}$ i.e. subject was not present in the RC during off-body measurements (section II-C1), an AA range of $0.05 \mathrm{~cm}^{2}$ to $2.94 \mathrm{~cm}^{2}$ is obtained for $2100-\mathrm{DL}$ and $900-\mathrm{DL}$, respectively. For the loaded RC, i.e. subject was present during off-body measurements, the AA has a range of $0.21 \mathrm{~cm}^{2}$ (2100-DL) to $15.44 \mathrm{~cm}^{2}$ (900-DL). A ratio of $4.6 \pm 0.53(\mu \pm \sigma)$ is obtained by dividing the AA values for the loaded RC by those of unloaded $\mathrm{RC}$. This ratio indicates that loading the $\mathrm{RC}$ with the subject increases the AA values and consequently decreases the actual measured incident fields in real indoor environment (equation 2) by a factor of up to 5. This is due to modification of the subject (the absence of the test person during the offbody measurements) that induces a random modification of the plane wave spectrum and results in variation of the RC's Qfactor [27]. In [12], using two PEMs calibrated in an unloaded $\mathrm{RC}$ resulted in higher measured $S_{i n c}$ (factor of 2.2) in an indoor environment compared to the measurements of the same PEMs calibrated in an anechoic chamber. Section III-C presents results of validation measurements of BWDM using both AA values in the unloaded and loaded RC. In this 
paper only standing posture is considered for calibration and validation measurements. In [26], deviations of $6 \%$ and $10 \%$ on the absorption cross section have been reported for a human subject in sitting position and with stretched arms, respectively, compared to when the subject was standing in a RC. This implies that calibration of BWDM with a different posture could result in small deviation of AA values due to increased or decreased body surface area (BSA) of the subject as a result of increased or decreased absorption of the EMFs in the body. In addition, the BSA of a subject depends on its height and weight [30]. The influence of the posture and body mass index of different subjects on the measurement uncertainty of the BWDM will be investigated in future research.

TABLE II

ANTENNA APERTURES (AA) OF BWDM OBTAINED FROM ON-BODY CALIBRATION IN LOADED AND UNLOADED REVERBERATION CHAMBER.

\begin{tabular}{|c|c|c|c|c|}
\hline \multirow{2}{*}{ Bands } & \multirow{2}{*}{$A A_{A N}^{\dagger}\left(\mathrm{cm}^{2}\right)$} & \multicolumn{2}{|c|}{$A A_{R C}\left(\mathrm{~cm}^{2}\right)$} & \multirow{2}{*}{$\begin{array}{c}\text { Ratio } \\
\text { loaded } \\
\text { unloaded }\end{array}$} \\
\hline & & unloaded & loaded & \\
\hline 800-DL & 9,82 & 1.84 & 8.36 & 4.5 \\
\hline 900-UL & 12,40 & 2.2 & 10.55 & 4.8 \\
\hline 900-DL & 12,72 & 2.94 & 15.44 & 5.2 \\
\hline 1800-UL & 4,36 & 1.82 & 9.33 & 5.1 \\
\hline 1800-DL & 2,59 & 1.58 & 8.04 & 5 \\
\hline DECT & 4,44 & 2.3 & 10.65 & 4.6 \\
\hline 2100-UL & 2,57 & 1.41 & 6.92 & 4.9 \\
\hline 2100-DL & 0,88 & 0.05 & 0.21 & 4.2 \\
\hline WiFi-2G & 2,69 & 1.94 & 8.9 & 4.6 \\
\hline 2600-DL & 2,92 & 1.65 & 7.26 & 4.4 \\
\hline WiFi-5G & 0.33 & 0.13 & 0.43 & 3.3 \\
\hline
\end{tabular}

${ }^{\dagger}$ AN: Anechoic chamber [16].

\section{B. Cross-talk}

Figure 6 depicts the on-body cross-talk $\left(C_{i j}\right)$ of BWDM and Expom measured in the RC. For the BWDM (Fig.6(a)), the highest cross-talk occurs for DECT when 1800-DL is emitted $(1 \mathrm{~dB})$ and for $1800-\mathrm{DL}$ when DECT is emitted $(-4 \mathrm{~dB})$. This has also been observed in literature [10], [11]. Since these are adjacent bands, selection of a narrow band filter is the design limitation of the BWDM. One solution could be the use of intermediate frequency (IF) filters to improve frequency selectivity. Additionally, in [31] post-hoc processing of the exposure data for detection and correction of cross-talk in three bands (1800-DL, DECT and 2100-UL) has been proposed.

When 2100-DL is emitted cross-talks of $-6 \mathrm{~dB}$ (factor of 3.9), -7.6 dB (factor of 5.75) and $-8 \mathrm{~dB}$ (factor of 6.3) are registered by $900-\mathrm{DL}, 1800-\mathrm{UL}$ and $800-\mathrm{DL}$, respectively. For the same emitted band, 1800-DL, DECT and 2100-UL registered a cross-talk of $-11 \mathrm{~dB}$ (factor of 12.5) while the rest of the bands registered a cross-talk of lower than $-14 \mathrm{~dB}$ (factor of 25.1). This is due to the wavelength at $2140 \mathrm{MHz}$ (2100-DL), which is comparable to the traces on the PCB of the BWDM nodes. Although the mentioned cross-talk is not large (compared to DECT), node shielding is required in order to reduce it. This will be investigated in a part of the future work. For the rest of the bands the cross-talk is in the range of $-20 \mathrm{~dB}$ to $-35 \mathrm{~dB}$ which is larger than a factor of 100 .

The on-body cross-talk of Expom is illustrated in Fig. 6(b). Similar to BWDM, the highest Expom cross-talk occurs for
DECT and 1800-DL. The 1800-DL registered a cross-talk of $-3.9 \mathrm{~dB}$ (factor of 2.4) when DECT was emitted while DECT registered $-14.7 \mathrm{~dB}$ (factor of 29.5) when 1800-DL was emitted. WiFi-5G registered cross-talks of $-6 \mathrm{~dB}$ (factor of 3.9), -7.6 dB (factor of 5.7), $-9 \mathrm{~dB}$ (factor of 7.9) and -11.2 dB (factor of 13.1), which corresponds to emitted 2100-UL/1800DL, 1800-UL/DECT, 2600-DL/2600-UL and 2100-DL/WiFi$2 \mathrm{G}$ bands, respectively. In comparison, the BWDM has a larger cross-talk than the Expom when 2100-DL is emitted due to unshielded nodes: $15.6 \mathrm{~dB}$ difference for 900-UL and limited to maximum $11.6 \mathrm{~dB}$ difference for other bands. Except 2100DL, for other bands, the BWDM has a $10 \mathrm{~dB}$ lower cross-talk than the Expom.

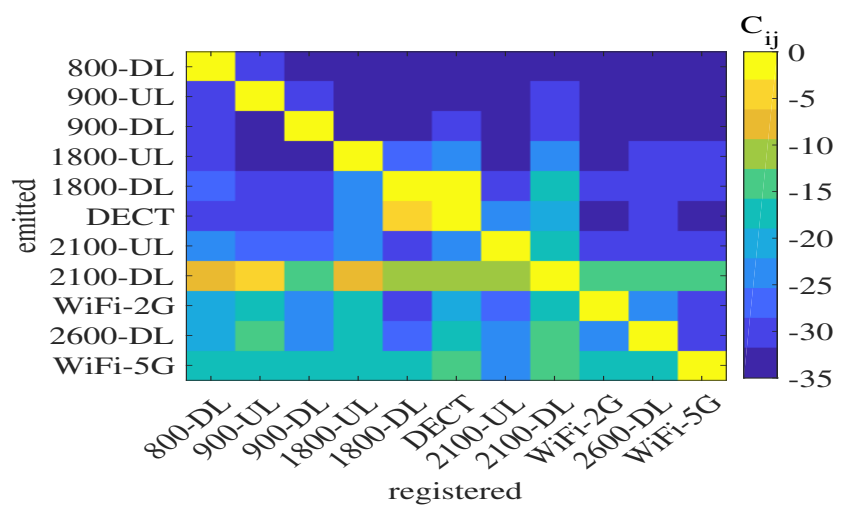

(a) BWDM

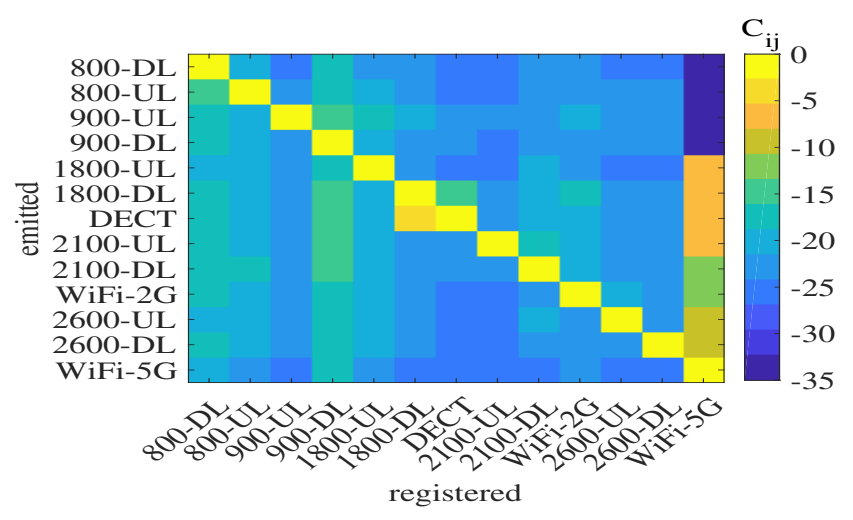

(b) Expom

Fig. 6. On-body cross-talk $\left(C_{i j}\right)$ of BWDM (a) and Expom (b) in the reverberation chamber.

\section{Validation in a real indoor environment}

Figure 7 shows the measured $S_{\text {inc }}$ using the BWDM and SA (the SA provides the true incident field values here) in five locations for five frequency bands. For the BWDM, three calibrations including AA values from the anechoic chamber, unloaded RC and loaded RC are applied (see equation 2).

Using the BWDM calibrated in the anechoic chamber, the difference between the measured $S_{i n c}$ by BWDM and SA are in the range of $0.5-1.5 \mathrm{~dB}, 0.2-3 \mathrm{~dB}, 3.2-8.1 \mathrm{~dB}, 7.1-15.9 \mathrm{~dB}$ and 3.4-16.2 $\mathrm{dB}$ for 800-DL, 900-DL, 1800-DL, 2100-DL and WiFi-2G, respectively.

The BWDM calibrated in the unloaded RC measured $S_{\text {inc }}$ values $5.7 \mathrm{~dB}$ to $8.1 \mathrm{~dB}$ higher than $\mathrm{SA}$ measurements for 800 - 
DL in five locations. For 900-DL the difference increases to $5.3 \mathrm{~dB}$ to $9.3 \mathrm{~dB}$ in different locations. Similar overestimation of $S_{i n c}$ up to $10.3 \mathrm{~dB}$ are observed for $1800-\mathrm{DL}$ w.r.t. the values registered by the SA. For 2100-DL, the BWDM measured values up to $14.5 \mathrm{~dB}$ higher than the SA. For WiFi$2 \mathrm{G}$, the BWDM underestimates $S_{\text {inc }}$ values by $2 \mathrm{~dB}$ to $14.8 \mathrm{~dB}$ lower than the SA.

The measured $S_{i n c}$ values using the BWDM calibrated in the loaded RC are only $0.1 \mathrm{~dB}$ to $1.6 \mathrm{~dB}$ different than those of SA for $800-\mathrm{DL}$ in five locations. This clearly indicates that the RC should be loaded for the calibration measurements. For 900-DL, this difference is limited to 0.1-2.1 dB in different locations. Similar differences between SA and BWDM calibrated in the loaded RC are observed for 1800DL: $1.6 \mathrm{~dB}$ to $3.2 \mathrm{~dB}$. For 2100-DL, the BWDM measured lower $S_{\text {inc }}(0.7 \mathrm{~dB})$ than SA for office 2 and $2.2 \mathrm{~dB}$ to $8 \mathrm{~dB}$ higher $S_{i n c}$ than SA for the other four locations. Although the difference between the SA and calibrated BWDM in loaded $\mathrm{RC}$ is up to $8 \mathrm{~dB}$ for $2100-\mathrm{DL}$, these differences are the lowest compared to the measured values using the BWDM calibrated in unloaded RC and anechoic chamber. For WiFi$2 \mathrm{G}$, the BWDM calibrated in the loaded RC measured lower $S_{i n c}$ than SA in the range of $8.6 \mathrm{~dB}$ to $21.3 \mathrm{~dB}$.

Figure 7 also demonstrates the underestimation of RMS values of incident fields in WiFi-2G band using three calibrations i.e. anechoic chamber, loaded and unloaded RC. However, it must be noted that the limitation for measuring WiFi-2G signals with the tri-axial antenna and SA is that the difference between DL and UL could not be distinguished. If during the measurements somebody had passed with a phone having an active WiFi connection (transmitting), the SA would measure the duty cycle in which the electric field was maximum. Therefore, for WiFi-2G, the calibration of BWDM in loaded $\mathrm{RC}$ is reprocessed with maximum value of $A A_{\text {geom }}$ $\left(41.18 \mathrm{~cm}^{2}\right)$ instead of averaging the $A A_{\text {geom }}$ values over all positions of the stirrer $\left(8.9 \mathrm{~cm}^{2}\right)$. Next, this maximum AA is applied to the maximum registered powers in $\mathrm{WiFi}-2 \mathrm{G}$ band by the BWDM during the validation measurements. For SA, maximum measured E-fields in $\mathrm{WiFi}-2 \mathrm{G}$ band is considered without applying the duty cycles. The results are shown in Fig. 7 (filled markers) and are compared in Table III.

Table III lists the difference of measured incident power densities $\left(\Delta_{S_{i n c}}\right)$ between the SA and BWDM calibrated in loaded RC. Considering the maximum AA in the loaded RC the maximum measured fields with the BWDM are in very good agreement with the maximum measured values of the $\mathrm{SA}$ in the WiFi-2G band. The difference between the two measurements is in the range of $0.6 \mathrm{~dB}$ to $2.37 \mathrm{~dB}$. Except for 2100-DL, the maximum difference between measurements of the BWDM and SA is limited to $3.23 \mathrm{~dB}$ (1800-DL in corridor 3). Differences up to $8 \mathrm{~dB}$ are obtained for 2100 DL but this might be attributed to the larger cross-talk values as discussed previously. Consequently, the difference for the total exposure between SA and BWDM is below $0.9 \mathrm{~dB}$ (corridor 1), which is an excellent agreement.

The characteristics of the BWDM calibrated in loaded RC are listed in Table IV. An AA range of $0.21 \mathrm{~cm}^{2}$ (2100-DL) to $41.18 \mathrm{~cm}^{2}(\mathrm{WiFi}-2 \mathrm{G})$ results in an on-body limit of detection
TABLE III

THE DIFFERENCE OF MEASURED INCIDENT POWER DENSITIES IN DOWNLINK BANDS BY BWDM CALIBRATED IN LOADED RC AND SPECTRUM ANALYZER.

\begin{tabular}{lcccccc}
\hline \multirow{2}{*}{ Locations } & \multicolumn{6}{c}{$\Delta_{S_{i n c}}=S_{i n c, B W D M}-S_{i n c, S A}$} \\
\cline { 2 - 7 } & $800 \mathrm{~L}$ & 900 & 1800 & 2100 & WiFi-2G $^{*}$ & Total $^{*}$ \\
\hline \hline Office 1 & 1.55 & 1.91 & 1.9 & 8.05 & -1.51 & -0.58 \\
Corridor 1 & -0.81 & -1.89 & 2.3 & 4.97 & -2.37 & -0.92 \\
Corridor 2 & 1.6 & 2.16 & 2.1 & 2.27 & 0.72 & 0.51 \\
Corridor 3 & -0.38 & -1.09 & 3.23 & 6.12 & -1.2 & -0.35 \\
Office 2 & 0.16 & -0.10 & -1.68 & -0.74 & 0.6 & 0.18 \\
\hline
\end{tabular}

* Maximum measured E-fields by spectrum analyzer (SA) vs. maximum AA in loaded RC applied to the maximum registered power by WiFi-2G nodes of the BWDM.

(LOD) in the range of $0.04 \mu W / m^{2}$ to $1.5 \mu W / m^{2}$ (WiFi$5 \mathrm{G})$ for different frequency bands. The LODs of BWDM are compared by those of the Expom (see Section II-B) in the corresponding frequency bands. Except 2100-DL and 2600DL, the BWDM has an LOD of 4.2 times (WiFi-5G) lower to 3.5 times (900-UL and DECT) higher w.r.t LOD of Expom. Although the BWDM has LOD values higher than those of a single Expom (for 9 bands), factors larger than 2 have been reported in literature [9], [11] for the underestimation of PEMs.

In [12], 50\% confidence intervals $\left(C I_{50}\right)$ of $2.2 \mathrm{~dB}$ to $3 \mathrm{~dB}$ have been reported for the average over two PEMs (EME Spy 140) on the front and back of the body calibrated in a RC. The calibrated BWDM in RC exhibits a similar $C I_{50}$ in the range of $2.1 \mathrm{~dB}$ to $3.6 \mathrm{~dB}$.

TABLE IV

CHARACTERISTICS OF BWDM CALIBRATED IN LOADED RC.

\begin{tabular}{|c|c|c|c|c|c|}
\hline \multirow{2}{*}{ Bands } & \multicolumn{3}{|c|}{ BWDM } & \multirow{2}{*}{$\begin{array}{l}2 \mathrm{PEMs}^{\dagger} \\
\mathrm{CI}_{50}(\mathrm{~dB})\end{array}$} & \multirow{2}{*}{$\begin{array}{c}\text { Expom } \\
\text { LOD }\left(\mu W / m^{2}\right)\end{array}$} \\
\hline & AA $\left(\mathrm{cm}^{2}\right)$ & $\operatorname{LOD}\left(\mu W / m^{2}\right)$ & $\mathrm{CI}_{50}(\mathrm{~dB})$ & & \\
\hline 800-DL & 8.36 & 0.11 & 3.2 & & 0.07 \\
\hline 900-UL & 10.55 & 0.23 & 3.1 & 2.8 & 0.07 \\
\hline 900-DL & 15.44 & 0.11 & 3.4 & 3 & 0.07 \\
\hline $1800-$-UL & 9.33 & 0.12 & 3 & 2.7 & 0.07 \\
\hline 1800-DL & 8.04 & 0.11 & 3.5 & 2.6 & 0.07 \\
\hline DECT & 10.65 & 0.23 & 3.6 & 3 & 0.07 \\
\hline 2100-UL & 6.92 & 0.07 & 3.3 & 2.8 & 0.02 \\
\hline 2100-DL & 0.21 & 0.21 & 3 & 2.5 & 0.02 \\
\hline $\mathrm{WiFi}-2 \mathrm{G}^{*}$ & 41.18 & 0.04 & 2.5 & 2.3 & 0.07 \\
\hline 2600-DL & 7.26 & 0.19 & 2.6 & - & 0.02 \\
\hline WiFi-5G & 0.43 & 1.55 & 2.1 & 2.2 & 6.63 \\
\hline
\end{tabular}

* Maximum AA in loaded RC.

$\dagger$ The average over two PEMs on the front and back of a subject [12] LOD: limit of detection; $\mathrm{CI}_{50}: 50 \%$ confidence interval.

\section{CONCLUSION}

In this paper we presented design and calibration of a multiband body-worn distributed exposure meter (BWDM) in a reverberation chamber (RC) for personal exposure assessment in indoor diffuse environments. The proposed BWDM consists of 22 nodes for 11 frequency bands. Using the geometric average over two nodes, the BWDM has an uncertainty in terms of the $68 \%$ confidence interval $\left(C_{68}\right)$ of its antenna apertures in the range of 3.4 to $5.5 \mathrm{~dB}$. The BWDM is less affected by the wearer's body and a maximum difference of $0.5 \mathrm{~dB}$ was obtained for the $C I_{68}$ by changing the location of the nodes in the RC for four bands (900-UL and 900DL, WiFi-2G and 2600-DL). The results showed that on-body 

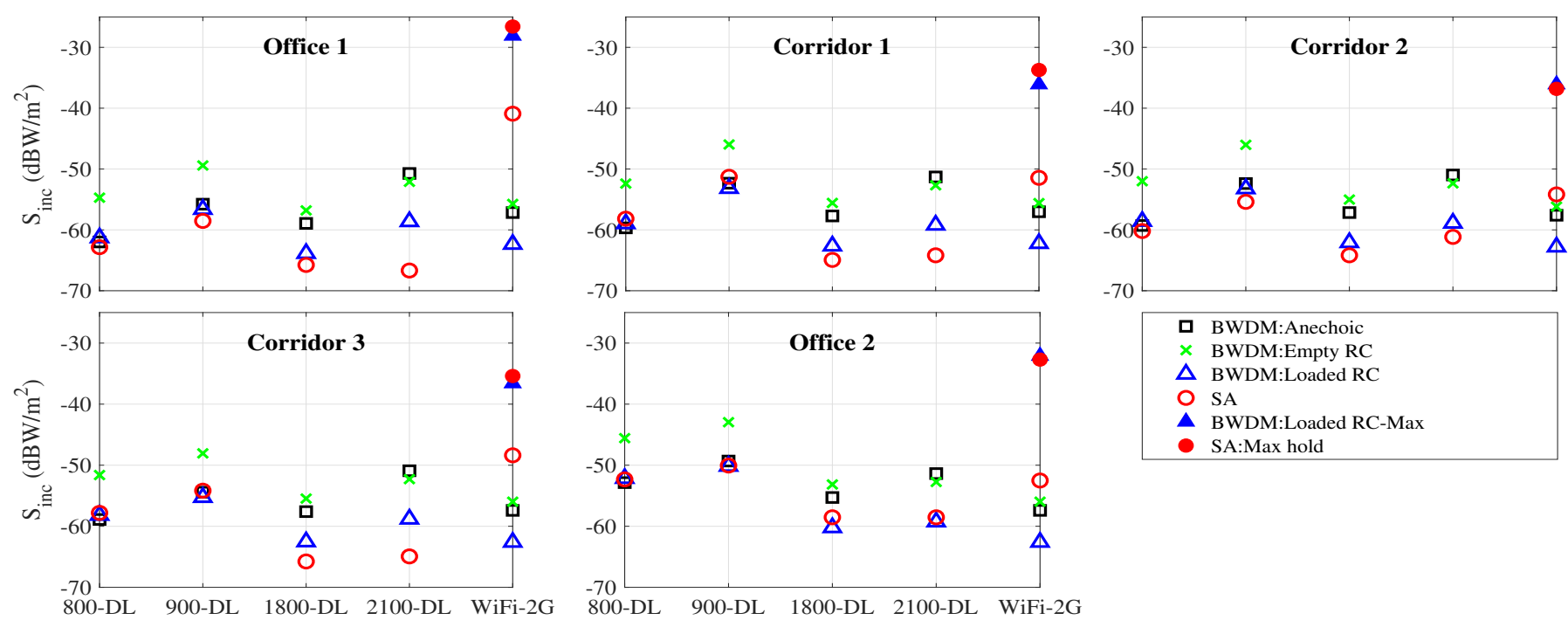
口 BWDM:Anechoic
$\triangle$ BWDM:Loaded RC
O SA
BWDM:Loaded RC-Max
BWDM:Loaded
SA:Max hold

Fig. 7. Comparison of $S_{i n c}$ measured by BWDM calibrated in an anechoic chamber, an unloaded and loaded reverberation chamber (RC) with $S_{\text {inc }}$ measured by spectrum analyzer (SA) in five locations for five frequency bands. For WiFi-2G, maximum measured $S_{i n c}$ by SA are shown vs. maximum AA in loaded $\mathrm{RC}$ applied to the maximum registered power by WiFi-2G nodes of the BWDM.

calibrations of the BWDM in an unloaded RC (i.e. subject was not present in the RC during off-body measurements) overestimated the incident fields up to a factor of 5. The results were validated using a tri-axial antenna and spectrum analyzer (SA) for five frequency bands in five indoor locations. The results of on-body calibration of the BWDM in the loaded RC (i.e. subject was present), were in very good agreement with SA measurements. Maximum differences up to $3.2 \mathrm{~dB}$ were obtained for the BWDM compared to the SA for four out of five bands in five locations. A maximum difference of $0.9 \mathrm{~dB}$ was obtained between measurements of the BWDM and SA for the total exposure in five locations which is an excellent agreement. Based on the results, the authors suggest on-body calibration of (commercial, distributed, wearable) PEMs to be performed in a loaded RC (e.g. in presence of a human-body phantom) instead of an empty RC. The on-body cross-talk was measured for the BWDM and an Expom in the RC. The results showed a high cross-talk for 1800-DL and DECT bands for both devices. One potential solution for the BWDM would be selection of narrow-band IF (intermediate frequency) filters for these bands to improve the frequency selectivity. In addition, compared to DECT, smaller cross-talk values were observed for the BWDM by other frequency bands (i.e. registering a small portion of $2100-\mathrm{DL}$ band). This might be due to the comparable wavelength in 2100-DL band with the traces on the printed circuit board of the nodes. This could be reduced by shielding the nodes which is part of the future work. Except 2100-DL, for other bands, the BWDM has a $10 \mathrm{~dB}$ lower cross-talk than the Expom. The influence of subjects' posture as well as body mass index of different subjects on the measurement uncertainty of the BWDM will be studied in future research.

\section{REFERENCES}

[1] World Health Organization (WHO), "Radio frequency (RF) fields research agenda," http://www.who.int/peh-emf/research/agenda/en/, ac- cessed on 15 February 2019

[2] M. Foerster, A. Thielens, W. Joseph, M. Eeftens, and M. Röösli, “A Prospective Cohort Study of Adolescents' Memory Performance and Individual Brain Dose of Microwave Radiation from Wireless Communication," Environmental Health Perspectives, vol. 126, no. 7, p. 077007, Jul. 2018.

[3] International Commission on Non-ionizing Radiation Protection (ICNIRP), "Guidelines for limiting exposure to time-varying electric, magnetic, and electromagnetic fields (up to $300 \mathrm{GHz}$ )," Health Phys., vol. 74, no. 4, pp. 494-522, 1998.

[4] S. Sagar, B. Struchen, V. Finta, M. Eeftens, and M. Röösli, "Use of portable exposimeters to monitor radiofrequency electromagnetic field exposure in the everyday environment," Environmental Research, vol. 150, pp. 289-298, Oct. 2016.

[5] M. Röösli, P. Frei, E. Mohler, C. Braun-Fahrländer, A. Bürgi, J. Fröhlich, G. Neubauer, G. Theis, and M. Egger, "Statistical analysis of personal radiofrequency electromagnetic field measurements with nondetects," Bioelectromagnetics, vol. 29, no. 6, pp. 471-478, 2008.

[6] W. Joseph, P. Frei, M. Roösli, G. Thuróczy, P. Gajsek, T. Trcek, J. Bolte, G. Vermeeren, E. Mohler, P. Juhász, V. Finta, and L. Martens, "Comparison of personal radio frequency electromagnetic field exposure in different urban areas across europe," Environmental Research, vol. 110, no. 7 , pp. $658-663,2010$.

[7] S. Mann, "Assessing personal exposures to environmental radiofrequency electromagnetic fields," Comptes Rendus Physique, vol. 11, no. 9, pp. 541-555, 2010.

[8] J. F. B. Bolte, "Lessons learnt on biases and uncertainties in personal exposure measurement surveys of radiofrequency electromagnetic fields with exposimeters," Environment International, vol. 94, pp. 724-735, Sep. 2016.

[9] J. F. Bolte, G. van der Zande, and J. Kamer, "Calibration and uncertainties in personal exposure measurements of radiofrequency electromagnetic fields," Bioelectromagnetics, vol. 32, no. 8, pp. 652-663, 2011.

[10] G. Neubauer, S. Cecil, W. Giczi, B. Petric, P. Preiner, J. Fröhlich, and M. Röösli, "The association between exposure determined by radiofrequency personal exposimeters and human exposure: A simulation study," Bioelectromagnetics, vol. 31, no. 7, pp. 535-545, 2010.

[11] A. Thielens, S. Agneessens, L. Verloock, E. Tanghe, H. Rogier, L. Martens, and W. Joseph, "On-body calibration and processing for a combination of two radio-frequency personal exposimeters," Radiation Protection Dosimetry, vol. 163, no. 1, pp. 58-69, 2015.

[12] R. Aminzadeh, A. Thielens, A. Bamba, L. Kone, D. P. Gaillot, M. Lienard, L. Martens, and W. Joseph, "On-body calibration and measurements using personal radiofrequency exposimeters in indoor diffuse and specular environments," Bioelectromagnetics, vol. 37, no. 5, pp. 298-309, 2016.

[13] A. Thielens, H. De Clercq, S. Agneessens, J. Lecoutere, L. Verloock, 
F. Declercq, G. Vermeeren, E. Tanghe, H. Rogier, R. Puers, L. Martens, and W. Joseph, "Personal distributed exposimeter for radio frequency exposure assessment in real environments," Bioelectromagnetics, vol. 34 no. 7, pp. 563-567, 2013.

[14] A. Thielens, S. Agneessens, H. De Clercq, J. Lecoutere, L. Verloock, E. Tanghe, S. Aerts, R. Puers, H. Rogier, L. Martens, and W. Joseph, "On-body calibration and measurements using a personal, distributed exposimeter for wireless fidelity," Health Phys, vol. 108, no. 4, pp. 407$418,2015$.

[15] P. Vanveerdeghem, P. Van Torre, A. Thielens, J. Knockaert, W. Joseph, and H. Rogier, "Compact personal distributed wearable exposimeter,' IEEE Sensors Journal, vol. 15, no. 8, pp. 4393-4401, 2015.

[16] R. Aminzadeh, A. Thielens, S. Agneessens, P. Van Torre, M. Van den Bossche, S. Dongus, M. Eeftens, A. Huss, R. Vermeulen, R. de Seze, P. Mazet, E. Cardis, H. Rogier, M. Röösli, L. Martens, and W. Joseph, "A multi-band body-worn distributed radio-frequency exposure meter: Design, on-body calibration and study of body morphology," Sensors, vol. 18 , no. 272,2018

[17] K. Kalliola, K. Sulonen, H. Laitinen, O. Kivekas, J. Krogerus, and P. Vainikainen, "Angular power distribution and mean effective gain of mobile antenna in different propagation environments," IEEE Transactions on Vehicular Technology, vol. 51, no. 5, pp. 823-838, Sep. 2002.

[18] C. Aliaga, "How is the time of women and men distributed in europe. statistics in focus, population and social conditions. issn 1024-4352, ks-nk-06-004-en-n, eurostat Luxembourg,' 2006, http://www.unece.org/fileadmin/DAM/stats/gender/timeuse/ DataReports/How_is_the_time_of_Women_and_Men.pdf, accessed on 15 February 2019

[19] J. B. Andersen, J. O. Nielsen, G. F. Pedersen, G. Bauch, and J. M Herdin, "Room electromagnetics," IEEE Antennas and Propagation Magazine, vol. 49, no. 2, pp. 27-33, April 2007.

[20] J. B. Andersen, K. L. Chee, M. Jacob, G. F. Pedersen, and T. Kurner, "Reverberation and absorption in an aircraft cabin with the impact of passengers," IEEE Transactions on Antennas and Propagation, vol. 60 no. 5, pp. 2472-2480, May 2012.

[21] J. Poutanen, J. Salmi, K. Haneda, V. M. Kolmonen, and P. Vainikainen, "Angular and shadowing characteristics of dense multipath components in indoor radio channels," IEEE Transactions on Antennas and Propagation, vol. 59, no. 1, pp. 245-253, Jan 2011

[22] A. Bamba, W. Joseph, G. Vermeeren, E. Tanghe, D. P. Gaillot, J. B. Andersen, J. o. Nielsen, M. Lienard, and L. Martens, "Validation of experimental whole-body sar assessment method in a complex indoor environment," Bioelectromagnetics, vol. 34, no. 2, pp. 122-132, 2013.

[23] P. Besnier and B. Démoulin, Electromagnetic Reverberation Chambers, 1st ed. Hoboken, USA: Wiley-ISTE, Feb. 2013. [Online]. Available: https://onlinelibrary.wiley.com/doi/book/10.1002/9781118602034

[24] J. Lecoutere, A. Thielens, S. Agneessens, H. Rogier, W. Joseph, and R. Puers, "Wireless Fidelity Electromagnetic Field Exposure Monitoring With Wearable Body Sensor Networks," IEEE Transactions on Biomedical Circuits and Systems, vol. 10, no. 3, pp. 779-786, Jun. 2016.

[25] S. Agneessens, "Coupled Eighth-Mode Substrate Integrated Waveguide Antenna: Small and Wideband With High-Body Antenna Isolation,' IEEE Access, vol. 6, pp. 1595-1602, 2018.

[26] A. Bamba, D. P. Gaillot, E. Tanghe, G. Vermeeren, W. Joseph, M. Lienard, and L. Martens, "Assessing whole-body absorption cross section for diffuse exposure from reverberation chamber measurements," IEEE Transactions on Electromagnetic Compatibility, vol. 57, no. 1, pp. 27 34, Feb 2015.

[27] R. Aminzadeh, A. K. Fall, J. Sol, A. Thielens, P. Besnier, M. Zhadobov, N. De Geeter, P. P. Vasudevan, L. Dupré, R. Van Holen, L. Martens, and W. Joseph, "Design and Calibration of a mm-Wave Personal Exposure Meter for 5g Exposure Assessment in Indoor Diffuse Environments,' Journal of Infrared, Millimeter, and Terahertz Waves, vol. 39, no. 12 pp. 1264-1282, Dec. 2018 .

[28] CENELEC (European Committee for Electrotechnical Standardization), 2008. TC106x WG1 EN 50492 in situ, "Basic standard for the in-situ measurement of electromagnetic field strength related to human exposure in the vicinity of base stations. Brussels, Belgium," 2008.

[29] W. Joseph, L. Verloock, F. Goeminne, G. Vermeeren, and L. Martens, "Assessment of RF exposures from emerging wireless communication technologies in different environments," Health Physics, vol. 102, no. 2, pp. 161-172, Feb. 2012.

[30] E. H. Livingston and S. Lee, "Body surface area prediction in normal-weight and obese patients," American Journal of PhysiologyEndocrinology and Metabolism, vol. 281, no. 3, pp. E586-E591, Sep. 2001.
[31] M. Eeftens, B. Struchen, K. Roser, M. Zahner, J. Fröhlich, and M. Röösli, "Dealing with crosstalk in electromagnetic field measurements of portable devices," Bioelectromagnetics, vol. 39, no. 7, pp. 529538, Oct. 2018.

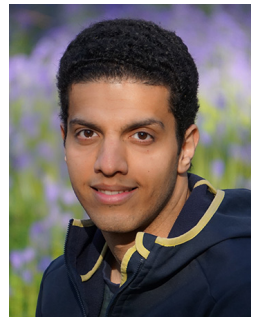

Reza Aminzadeh (S'10) received the M.Sc. degree in electrical engineering from Sharif University of Technology, Iran, in 2014. Since 2015, he is with the Department of Information Technology (INTEC) Ghent University-imec, Belgium, where, he is working toward a $\mathrm{PhD}$ degree mainly focused on wireless body area networks for characterization of human exposure to radio-frequency electromagnetic fields. From 2012 to 2014, he was a researcher in the computational electromagnetics lab at Sharif University of Technology, where he conducted research on numerical and experimental assessment of millimeter-wave reflectometry for detection of skin cancer. Reza Aminzadeh was served as a member of the technical program committee for the $14^{t h}, 15^{t h}$ and $17^{t h}$ National Iranian Student Conference on Electrical Engineering (ISCEE) during 2011-2014. He is the author or co-author of about 24 peer-reviewed scientific journals and conference papers in bioelectromagnetics.

Reza Aminzadeh is the recipient of the third Best Platform Presentation and Scientific Paper Award in BioEM2018, the Joint Meeting of The Bioelectromagnetics Society (BEMS) and the European BioElectromagnetics Association (EBEA). His research interests include numerical and experimental dosimetry, measurement and theoretical modeling of dielectric properties of biological tissues, development of tissue-equivalent phantoms for the mm-wave band including the $5 \mathrm{G}$ technology and on/in body radio wave propagation.

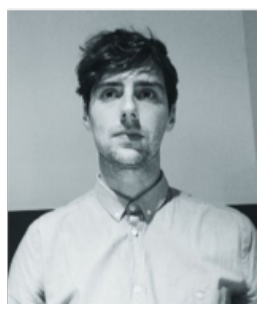

Arno Thielens graduated in September 2010 from Ghent University as a master of science in engineering: applied physics. In the same year, he joined the Waves group, at that time known as $\mathrm{WiCa}$, of Ghent University's information technology (INTEC) department as a PhD student. His research focused on personal exposure assessment to radio-frequency electromagnetic fields and numerical dosimetry. He obtained a PhD in Applied Physics at Ghent University in May 2015. Since June 2015, he is working as a post-doctoral researcher at Waves. He was a post-doctoral fellow of the IWT (Institute for Science and Innovation, Flanders, Belgium) from January 2016 up to May 2017. He obtained an FWO [PEGASUS] $^{2}$ Marie Skłodowska-Curie Fellowship in 2017 funded by the European Union's Horizon 2020 research and innovation programme under the Marie Skłodowska-Curie grant agreement No 665501 with the research Foundation Flanders (FWO). He also received an Honorary Fellowship by the Belgian American Education Foundation (BAEF) in the same year. In June 2017, he joined the Berkeley Wireless Research Center at the University of California, Berkeley, where he is working on the development of the human intranet. Dr. Thielens is the recipient of the Joseph James Morrissey Memorial Award issued by the Bioelectromagnetics Society (BEMS) and the European Bio Electromagnetics Association (EBEA) in June 2013. He also received the 2015 International Union of Radio Science (Union Radio-Scientifique Internationale or URSI) young scientist award. 


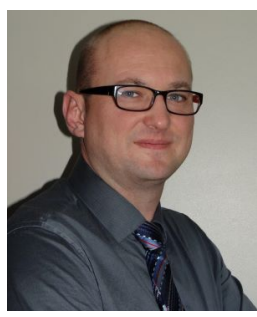

Davy P. Gaillot was born in Arras, France, on October 26, 1978. He received the M.Sc. degree in mechanical engineering from Ecole Nationale d'Ingénieurs de Metz (ENIM), Metz, France, in 2002, the M.Sc. degree in mechanics, materials, structures, and processes from the University of Metz, Metz, France, in 2002 and the Ph.D. degree in materials science and engineering from the Department of Materials Science and Engineering, Georgia Institute of Technology, Atlanta, GA, USA, in 2007. Since 2008, he has been an Associate Professor with the University of Lille 1 in the IEMN/TELICE Group (Institut d'Electronique, de Microélectronique et de Nanotechnologie/Télécommunications, Interférences et Compatibilité Electromagnétique), Villeneuve d'Ascq, France. His research interests include the development of outdoor/indoor radio channel models for localization techniques that are supported by legacy or upcoming millimeter-wave and centimeter-wave wireless network standards. In addition, he focuses on highly diffuse and industrial radio channels for propagation modeling and exposure assessment.

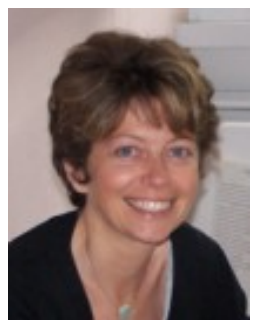

Martine Liénard received the Ph.D. degree from the University of Lille, Lille, France, in 1993. Since 1990, she has been with the Telecommunications, Interferences and Electromagnetic Compatibility (TELICE) group of the Institute of Electronic, Microelectronic and Nanotechnology (IEMN) of University of Lille. She is currently a Professor at University of Lille and Head of the TELICE Group. Her current research deals with mobile localization techniques in non-cooperative mode and theoretical and experimental channel characterization in complex environment. She is also involved in challenges of MIMO and Massive MIMO techniques, mainly dedicated for communication in rail and road transportation systems and industry 4.0.

Lamine Koné was born in Karangana, Mali, in 1956. He received the M.Sc and Ph.D. degrees from the University of Lille, Villeneuve d'Ascq, France, in 1985 and 1989, respectively, both in electronics. Since 1990, he has been a Research Engineer with the IEMN Laboratory, Telice Group, University of Lille. His current research interests include electromagnetic compatibility testing methods, coupling phenomena of EM field to cable assembly, various methods for measuring transfer impedance, and shielding effectiveness of cables and connectors.

Sam Agneessens photograph and biography not available at the time of submission.

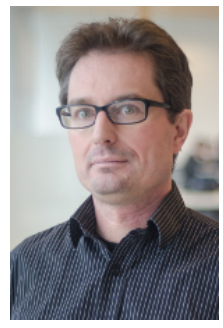

Patrick Van Torre $\mathrm{PhD}$, received the Electrical Engineering degree in 1995 and the doctoral degree at Ghent University, Belgium in 2012. He has been employed by Ghent University, at the Faculty of Engineering and Architecture since 1999, where he teaches theory courses in Electronics and ICT, organizes project-oriented lab sessions and is involved in hardware development projects for third parties. $\mathrm{He}$ is active as a researcher, in the field of wireless communication, focusing on body-centric multipleinput multiple-output (MIMO) and beam-forming systems as well as sub-GHz long range wireless sensor networks.

Matthias Van den Bossche graduated in 2013 from as Master of Electronics and ICT Engineering Technology from Leuven University (former Katholieke hogeschool Sint Lieven). In the same year, he joined the Waves research group after working in the group towards his master thesis on "Lone worker alarm systems". Here he works as a Technical and Research Assistant mainly focusing on propagation measurements, developing low cost measurement equipment and measurement procedures to access electromagnetic field exposure of emerging technologies (e.g. IoT, Smart meters, 4G/5G, WiFi, ...). Besides this he conducts the in-situ electromagnetic field measurements (by order of the government, telecom operators, other third parties) for compliance evaluation with the current regional and international guidelines.

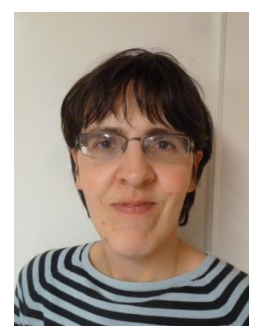

Leen Verloock was born in Eeklo, Belgium, on November 15, 1979. She received the Master'sdegree in electronics engineering from Katholieke Hogeschool Ghent, Ghent, Belgium, in 2001. From September 2001 to September 2005, she was with IMEC, Department of Information Technology(INTEC), Ghent University, where she was a Technical and Research Assistant in the Wireless \& Cable research group. Since October 2005, she has been with Ghent University in the same research group and continues her work as before. She is working on propagation effects for wireless systems, including the influence of the user on this propagation, and on measurement procedures to assess electromagnetic field exposure of new wireless technologies (e.g. LTE, 5G NR). Furthermore she is responsible for the in-situ electromagnetic field measurements (by order of the government, telecommunication operators, companies,...) for compliance evaluation with regional and international guidelines. She also assists Ph.D. students with their research on modeling and measuring the propagating fields of wireless systems.

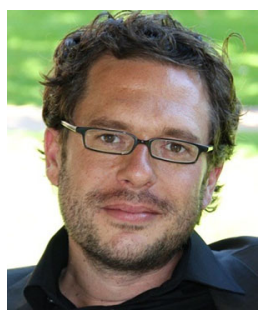

Stefan Dongus is a health geographer and epidemiologist based at the Swiss Tropical and Public Health Institute in Basel, Switzerland, where he received his $\mathrm{PhD}$ degree in 2009. Stefan Dongus is the scientific coordinator of the Swiss expert group on electromagnetic fields and non-ionising radiation (BERENIS), and a scientific collaborator at the Environmental Exposures and Health Unit, Department of Epidemiology and Public Health. Further research interests include geographic information systems (GIS) and mapping tools for operational use in disease control programmes. 


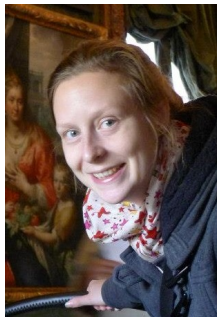

Marloes Eeftens is a Senior Scientific Collaborator in the Environmental Exposures and Health Unit at Swiss TPH, Basel, Switzerland. Before joining Swiss TPH in 2013, she completed a PhD in Toxicology and Environmental Health at the Institute of Risk Assessment Sciences in Utrecht, the Netherlands. As an environmental epidemiologist, she specializes in the effects of environmental exposures on respiratory, cardiovascular and cognitive health Marloes has a keen interest in new data collection tools for environment and health data, such as personal measurement devices, mobile phone apps, wearables and textileintegrated sensors. She has worked extensively on measurements and spatial modelling of environmental exposures: radiofrequency electromagnetic fields, air pollutants, noise and airborne pollen.

Anke Huss is an assistant professor in environmental epidemiology with a special focus on non-ionizing radiation research. She is involved in advanced methods of exposure assessment to RF-EMF including modelling of these exposures from base stations and has previously evaluated possible effects of from the exposure to electromagnetic fields on a range of health outcomes including well-being, sleep quality, neurodegenerative diseases and cancer using different study designs such as case-control and cohort studies.

Roel Vermeulen photograph and biography not available at the time of submission.

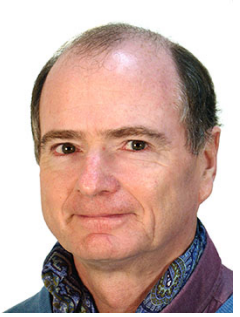

René de Seze MD, $\mathrm{PhD}$ has a background in physics and specialized in radiology (MRI). His research interests address the biological effects of electromagnetic fields. His doctoral research on the effects of microwaves on the murine immune system was performed in the Bioelectromagnetics Laboratory of Bernard Veyret in Bordeaux. He also looked at the therapeutic effects of magnetic fields on cancer in mice and rats. He then moved to the Medical Biophysics Department at the Medical School of Montpellier-Nîmes. He conducted studies on health risks of mobile phones: in human laboratory studies, on the endocrine system, on the auditory function, on neurophysiology and cognitive performances, on heat exchanges from the phone and/or the radiofrequency (RF) exposure; on the nervous system of rats. René de SEZE is now researcher at the National Institute of Industrial Environment and RISks (INERIS) in Verneuil en Halatte close to Paris. He developed there his experience on health impact of electromagnetic fields and continues to manage studies on this topic in the department of chronic risks, toxicology unit. He is involved in studies on effects of RF on thermoregulation in rodents. He is now Presidentelect of the Bioelectromagnetics Society (BEMS), member of the Sociéte Française de Radioprotection (SFRP) and has been President of the European Bioelectromagnetics Association (EBEA).

Elisabeth Cardis photograph and biography not available at the time of submission.

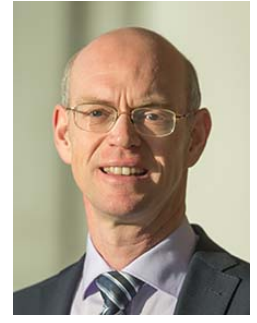

Hendrik Rogier (SM'06) received the Electrical Engineering and the Ph.D. degrees from Ghen University, Gent, Belgium, in 1994 and in 1999 , respectively. He is a currently a Full Professor with the Department of Information Technology, Ghent University, a Guest Professor with the Interuniversity Microelectronics Centre, Ghent, Belgium, and a Visiting Professor with the University of Buckingham, Buckingham, U.K. From October 2003 to April 2004, he was Visiting Scientist at the Mobile Communications Group of Vienna University of Technology. He authored and coauthored about 155 papers in internationa journals and about 180 contributions in conference proceedings. His current research interests are antenna systems, radiowave propagation, body-centric communication, numerical electromagnetics, electromagnetic compatibility and power/signal integrity. He was twice awarded the URSI Young Scientist Award. Moreover, he received the 2014 Premium Award for Best Paper in IET Electronics Letters and several awards at conferences. He is an Associate Editor of IET Electronics Letters, IET Microwaves, Antennas and Propagation, and the IEEE Transactions on Microwave Theory and Techniques. He acts as the URSI Commission B representative for Belgium and is a Senior Member of the IEEE.

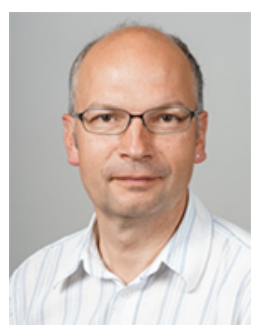

Martin Röösli received a M.Sc. degree in Environmental Science in from the Swiss Federal Institute of Technology in Zürich (ETHZ) in 1997 and a $\mathrm{Ph} . D$. in Epidemiology from the University of Basel in 2001. To date he is Professor for Environmental Epidemiology at the Swiss Tropical and Public Health Institute in Basel and leads the Environmental Exposures and Health Unit. His research focuses on various environmental health topics including ionizing and non-ionizing radiation, transportation noise, climate change, pesticides, passive smoking and ambient air pollution. He is conducting exposure assessment studies, aetiological research and health impact assessments. He is also member in various national and international commissions on environmental health risks including BERENIS (Swiss Expert Group on Non-Ionising Radiation), ICNIRP (International Commission for Non-ionizing Radiation Protection), the Scientific Council on Electromagnetic Fields of the Swedish Radiation Safety Authority, and Scientific Council of the European Bioelectromagnetics Association EBEA (2009 and 2017). He has published numerous scientific papers, reviews and book chapters (h-factor: 35 , Web of Science).

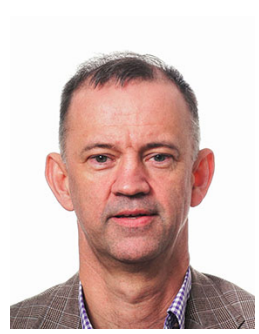

Luc Martens (M'92) received the M.Sc. degree in electrical engineering from Ghent University, Ghent, Belgium, in 1986, and the Ph.D. degree, in 1990. From 1986 to 1990 , he was a Research Assistant with the Department of Information Technology, Ghent University. During this period, his scientific research focused on the physical aspects of hyperthermic cancer therapy. His research dealt with electromagnetic and thermal modeling, and the development of measurement systems for that application. Since 1991, he has been managing the WAVES Research Group, INTEC. The WAVES Research Group is part of the imec Institute, since 2004. Since 1993, he has been a Professor with Ghent University. He has authored or co-authored more than 300 publications in the domain of electromagnetic channel predictions, dosimetry, exposure systems and health, and wireless communications. His research interests include modeling and measurement of electromagnetic channels, in electromagnetic exposure, e.g., around telecommunication networks and systems such as cellular base station antennas and in energy consumption of wireless networks. 


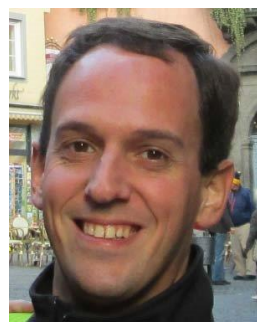

Wout Joseph (M'05-SM'12) was born in Ostend, Belgium, in 1977. He received the M.Sc. and Ph.D. degrees in electrical engineering from Ghent University, Ghent, Belgium, in 2000 and 2005, respectively. From 2000 to 2005, he was a Research Assistant with the Department of Information Technology, Ghent University. During this period, his scientific research focused on electromagnetic exposure assessment. Since 2005, he has been a Postdoctoral Researcher with INTEC, UGent-imec. Since 2009 he has been a Professor in the domain of experimental characterization of wireless communication systems. His research interest include measuring and modeling electromagnetic fields around base stations for mobile communications, health effects of exposure to electromagnetic radiation, electromagnetic exposure assessment, propagation for wireless communication systems, and antennas and calibration. He is specialized in wireless performance analysis and quality of experience. From 2007 to 2013 he was a Postdoctoral Fellow of the FWO-V (Research Foundation-Flanders). 